\title{
Feasibility of finite renormalization of particle mass in quantum electrodynamics
}

\author{
A.V. Gichuk,V.P. Neznamov, Yu.V. Petrov \\ Russian Federal Nuclear Center, Sarov, Russia, 607190
}

1st November 2018

\begin{abstract}
The paper proposes an algorithm for regularization of the self-energy expressions for a Dirac particle that meets the relativistic and gauge invariance requirements.

Within the second order formulations of the "old" perturbation theory for free motion, the expression for the upper integration limit $q$ is a slowly enough varying function of particle impulse. For a particle at rest, $q=m$; for $p / m$ in the range from 0 to 1 ,$$
q \approx m\left(1+0.00864 \frac{p^{4}}{m^{4}}-0.00217 \frac{p^{6}}{m^{6}}\right)
$$

for the ultra-relativistic case $|\mathbf{p}| \gg m: \quad q \approx 1.5 m$.

For $4 \mathrm{D}$ perturbation theory, on introduction of the limiting 4-impulse, $L^{2}=L_{0}^{2}-\mathbf{L}^{2}$, it is shown that with a large time component, $L_{0} / m \gg 1$, the spatial values of $L_{i}$ are limited and are the same as the components of the introduced limits of integration $q_{i}: \mathbf{L}^{2}=\mathbf{q}^{2}$.

Within the proposed algorithm, in the second-order of the perturbation theory, the renormalized Dirac particle mass is

$$
m^{(2)}=m_{0}+\Delta m^{(2)}=m_{0}\left(1+1.115 \frac{e^{2}}{\pi}\right),
$$

where $m_{0}$ is the bare mass of the particle.

The electromagnetic radius of particle at rest determined by equating the self- energy and the electrostatic energy $e^{2} / r_{e m}$ is about half the Compton wavelength, $r_{e m} \approx 1 / 2 m$.
\end{abstract}


It is commonly known that the calculation of some physical effects results in undefined diverging expressions, when the perturbation theory formalism of existing formulations of field and particle quantum theories is used. If the quantum-field theories are renormalizable, the undefined expressions are removed in all orders of the perturbation theory by renormalizing mass and charge of relevant particles. In particular, quantum electrodynamics is such a theory.

In the lifetime of the relativistic quantum-field theories, many researchers tried to solve the regularization problem of infinite expressions appearing in the computations from various viewpoints. The problem, however, remains unsolved.

This paper suggests, as its authors believe, a natural way for solving the problem of finite renormalization of particle mass by the example of quantum elecrodynamics.

First, use the formulations from the "old" perturbation theory given by Dirac for quantum electrodynamics in [1].

The self-energy operator in the second order of the perturbation theory is:

$$
Y=\frac{e^{2}}{\pi} \frac{1}{4 \pi} \int \alpha_{\mu} \frac{\nu+\tilde{E} /|\tilde{E}|}{|\tilde{E}|+|\mathbf{k}|-\nu E_{i}} \alpha^{\mu} \frac{d \mathbf{k}}{|\mathbf{k}|}
$$

In (11) and hereinafter $\hbar=c=1 ; \quad \alpha^{\mu}\left\{\begin{array}{l}1 \\ \alpha^{i}\end{array}\right.$ $\alpha^{i}, \beta$ are Dirac matrices; $\tilde{\mathbf{p}}=\mathbf{p}+\mathbf{k}$;

$$
\begin{array}{ll}
E=\alpha \pi+\beta m+e A_{0} ; & \pi^{i}=p^{i}-e A^{i} ; \quad \tilde{\pi}^{i}=p^{i}+k^{i}-e A^{i} \\
\tilde{E}=\alpha \tilde{\pi}+\beta m+e A_{0} ; & p_{0}=\left(m^{2}+\mathbf{p}^{2}\right)^{1 / 2} ; \quad \tilde{p}_{0}=\left(m^{2}+\mathbf{p}^{2}+2 \mathbf{p k}+\mathbf{k}^{2}\right)^{1 / 2}
\end{array}
$$

$A_{0}, A^{i}$ are scalar and vector potentials of the external electromagnetic field.

Expression (11) implies the averaging over $\nu:(1 / 2)(Y(\nu=1)+Y(\nu=-1))$.

In action on state $|i\rangle$ with energy $E_{i}$,

$$
E|i\rangle=E_{i}|i\rangle, \quad Y|i\rangle=Y_{i}|i\rangle
$$

Dirac considered three modes for the regularization of expression (11) in [1].

Mode $a$. Modulus of $\mathbf{k}$ is limited, $|\mathbf{k}|<q$, where $q$ is some number.

$\underline{\text { Mode } b}$. Here the sum of energy moduli of three particles involved in the interaction event is limited, $|\tilde{E}|+|\mathbf{k}|+\left|E_{i}\right|<2 q$.

Mode $c$. Here the sum of kinetic energies of three particles is limited,

$$
\left|\tilde{E}-e A_{0}\right|+|\mathbf{k}|+\left|E_{i}-e A_{0}\right|<2 q .
$$

For large $q$, the correspondence is established:

$$
\begin{aligned}
& Y_{a}=Y_{b}+\frac{1}{6} \alpha \pi+\frac{1}{2} e A_{0} ; \\
& Y_{a}=Y_{c}+\frac{1}{6} \alpha \pi
\end{aligned}
$$


As a result, Dirac prefers mode $c$ as a technique allowing us to eventually obtain relativisticallyand gauge-invariant expressions for the self-energy and vacuum polarization. As this is always the case in the relativistic quantum field theories, the numerical value of $q$ is not determined.

This paper uses mode $a$ to demonstrate the possibility to obtain the relativistically- and gauge-invariant expression for the self-energy with simultaneous estimation of the upper integration limit $q$.

First, consider the case where there are no external electromagnetic fields $\left(A_{0}=0, A^{i}=0\right)$. Expression (11) becomes

$$
Y_{0}=\frac{e^{2}}{4 \pi^{2}} \int \alpha_{\mu} \frac{\nu \tilde{p}_{0}+\alpha \tilde{\mathbf{p}}+\beta m}{\tilde{p}_{0}\left(\tilde{p}_{0}+|\mathbf{k}|-\nu p_{0}\right)} \alpha^{\mu} \frac{d \mathbf{k}}{|\mathbf{k}|} .
$$

Consider a non-relativistic charged particle having impulse $|\mathbf{p}| \ll m$.

On summation over $\mu$ and integration over azimuthal angle $\varphi$, we obtain

$$
Y_{0}=\frac{e^{2}}{\pi} \int_{0}^{q} \int_{-1}^{1} \frac{-\nu \tilde{p}_{0}+\alpha(\mathbf{p}+\mathbf{k})+2 \beta m}{\tilde{p}_{0}\left(\tilde{p}_{0}+|\mathbf{k}|-\nu p_{0}\right)} k d(\cos \theta) d k .
$$

Expanding the integrand in (5) over the particle impulse $|\mathbf{p}|$ up to quadratic expressions $\mathbf{p}^{2} / m^{2}$ and integrating give:

$$
\begin{aligned}
Y_{0}= & \frac{2 e^{2}}{\pi}\left\{m\left[-\frac{\ln (x)}{4}+\frac{1}{8} \frac{m^{2}}{x^{2}}+\frac{\mathbf{p}^{2}}{2 m^{2}}\left(\frac{1}{3} \frac{m^{2}}{x^{2}+1}-\frac{\ln (x)}{4}-\frac{1}{24} \frac{m^{2}}{x^{2}}\right)\right]\right. \\
& +\alpha \mathbf{p}\left(\frac{\ln (x)}{4}-\frac{m^{2}}{3\left(x^{2}+1\right)}-\frac{1}{24} \frac{m^{2}}{x^{2}}\right) \\
& \left.+\beta m\left[\ln (x)+\frac{\mathbf{p}^{2}}{2 m^{2}}\left(-\frac{4}{3} \frac{m^{2}}{x^{2}+1}+\frac{4 m^{2}}{\left(x^{2}+1\right)^{2}}-\frac{8}{3} \frac{m^{6}}{\left(x^{2}+1\right)^{3}}\right)\right]\right\}\left.\right|_{m} ^{A \cdot m}, \\
& x=k+\sqrt{m^{2}+k^{2}}, \quad A \cdot m=q+\sqrt{m^{2}+q^{2}} .
\end{aligned}
$$

Use equalities

$$
\left(-m-\frac{\mathbf{p}^{2}}{2 m}+\alpha \mathbf{p}+\beta m\right)|i\rangle \approx 0, \quad\langle i|\alpha \mathbf{p}| i\rangle=\left\langle i\left|\frac{\mathbf{p}^{2}}{p_{0}}\right| i\right\rangle \approx\left\langle i\left|\frac{\mathbf{p}^{2}}{m}\right| i\right\rangle
$$

to obtain

$$
\begin{aligned}
\left\langle i\left|Y_{0}\right| i\right\rangle= & \frac{e^{2}}{\pi}\langle i|\left\{\beta m\left(\frac{3}{2} \ln (A)+\frac{1}{4 A^{2}}-\frac{1}{4}\right)\right. \\
& \left.+\frac{\mathbf{p}^{2}}{m}\left(-\frac{5}{3\left(A^{2}+1\right)}+\frac{4}{\left(A^{2}+1\right)^{2}}-\frac{8}{3\left(A^{2}+1\right)^{3}}+\frac{1}{6}\right)\right\}|i\rangle .
\end{aligned}
$$


Reasoning from the relativistic invariance condition for the self-energy operator $Y_{0}$, require that the coefficient of $\mathbf{p}^{2} / m$ in (6) vanish. The natural condition for the upper limit of integration $q$ in (5) follows herefrom:

$$
\begin{aligned}
& -\frac{5}{3\left(A^{2}+1\right)}+\frac{4}{\left(A^{2}+1\right)^{2}}-\frac{8}{3\left(A^{2}+1\right)^{3}}+\frac{1}{6}=0 \quad \text { or } \\
& \frac{q}{\left(q^{2}+m^{2}\right)^{1 / 2}}\left(\frac{q^{2}}{q^{2}+m^{2}}-\frac{1}{2}\right)=0 .
\end{aligned}
$$

The solution to equation (77) is $q= \pm m$, in addition to the trivial one, $q=0$.

The value $q \approx m$ means that the upper integration limit imposes the restriction on the distances. They should be longer than Compton wavelength of Dirac charged particle: $|\mathbf{x}| \geq$ $1 / m$, which is quite reasonable from the viewpoint of quantum mechanics.

The value $q \approx m$ for the upper integration limit is also reasonable in terms of "Zitterbewegung" effect for Dirac particle. In fact, if Dirac Hamiltonian is written in the form

$$
H_{D}=\alpha \pi+\beta m+e A_{0} ; \quad \pi=\mathbf{p}-e \mathbf{A}
$$

in the presence of external fields, then the operator of coordinate $x^{i}$ can be represented as follows [2]:

$$
x^{i}=x_{0}^{i}+\pi^{i} \frac{1}{H_{D}-e A_{0}} t-\frac{1}{4} \alpha_{0}^{i} e^{-2 i\left(H_{D}-e A_{0}\right) t} \frac{1}{\left(H_{D}-e A_{0}\right)^{2}},
$$

where $\alpha_{0}^{i}$ is the matrix integration constant.

The third term of $x^{i}$ operator highly oscillates with frequency $\sim 2 m$. This term can be represented as

$$
\Delta x^{i}=\frac{i}{2}\left(\alpha^{i}-\pi^{i} \frac{1}{H_{D}-e A_{0}}\right) \frac{1}{H_{D}-e A_{0}}
$$

It is seen from (8) that $\Delta x^{i} \sim 1 / m$, and from this standpoint it is quite natural to restrict the consideration to distances $|\mathbf{x}| \geq 1 / m$.

Note that when previously estimating the self-energy of the non-relativistic charged particle in the self-field electrodynamics in Foldy-Wouthuysen representation, one of the authors also came to the necessity of the finite upper integration limit with $\Lambda \approx m[3$.

Clear, the value $q \approx m$ obtained from the expansion of (5) to quadratic values $\mathbf{p}^{2} / m^{2}$ can vary when the following expansion terms are included. The value of $q$ can also change with external electromagnetic fields $A^{\mu}(\mathbf{x})$ introduced.

Consider the function

$$
\langle i|F(p, q, m)| i\rangle=\left\langle i\left|Y_{0}\right| i\right\rangle-\frac{e^{2}}{\pi}\left\langle i\left|\beta m\left(\frac{3}{2} \ln \left(A_{0}\right)+\frac{1}{4 A_{0}^{2}}-\frac{1}{4}\right)\right| i\right\rangle,
$$

where $A_{0}=\left.A\right|_{q=m}=1+\sqrt{2}$.

The second addend in (9) is relativistically invariant. In its form, the addend corresponds to the infinite renormalization term to be added to the particle mass in standard quantum electrodynamics, if the integration limit $q \rightarrow \infty$ in $A(q)$. 
Table 1: The upper integration limit $q(p)$

\begin{tabular}{c|cccccc}
\hline $\mathrm{p} / \mathrm{m}$ & 0 & 0.01 & 0.02 & 0.04 & 0.05 & 0.06 \\
$\mathrm{q} / \mathrm{m}$ & 1 & 1.000000000088 & 1.0000000014 & 1.000000023 & 1.000000055 & 1.00000011 \\
\hline $\mathrm{p} / \mathrm{m}$ & 0.07 & 0.08 & 0.09 & 0.1 & 0.2 & 0.5 \\
$\mathrm{q} / \mathrm{m}$ & 1.00000021 & 1.00000036 & 1.00000058 & 1.00000088 & 1.000014 & 1.000512 \\
\hline $\mathrm{p} / \mathrm{m}$ & 0.8 & 1 & 10 & 100 & 500 & 1000 \\
$\mathrm{q} / \mathrm{m}$ & 1.00297 & 1.00647 & 1.26716 & 1.448 & 1.48117 & 1.494 \\
\hline
\end{tabular}

The function $F(p, m, q)$ is the relativistically non-invariant expression that (with our approach) should vanish with an appropriate choice of the upper integration limit $q$.

Table 团 gives $q / m$ versus $p / m$, for which $F(p, m, q)=0$.

The following equalities were used in the numerical computations:

$$
\langle i|\beta m| i\rangle=\left\langle i\left|\left(\frac{m^{2}}{\left(m^{2}+\mathbf{p}^{2}\right)^{1 / 2}}\right)\right| i\right\rangle, \quad\langle i|\alpha \mathbf{p}| i\rangle=\left\langle i\left|\left(\frac{\mathbf{p}^{2}}{\left(m^{2}+\mathbf{p}^{2}\right)^{1 / 2}}\right)\right| i\right\rangle .
$$

The computed data shows that the upper integration limit $q$ is a slowly enough varying function of $p / m$. Within the range of $p / m$ values from 0 to 1 , the function is amenable to approximation by the polynomial expression

$$
q \approx m\left(1+0.00864 \frac{p^{4}}{m^{4}}-0.00217 \frac{p^{6}}{m^{6}}\right) .
$$

For the ultra-relativistic case of $|\mathbf{p}| \gg m$, the function approaches $q \approx 1.5 m$ asymptote.

Thus, when choosing the upper integration limit $q(p)$ according to Table 1 for free motion, the average value of the self-energy operator (4) is a relativistic invariant,

$$
\left\langle i\left|Y_{0}\right| i\right\rangle=\frac{e^{2}}{\pi}\left\langle i\left|\beta m\left(\frac{3}{2} \ln \left(A_{0}\right)+\frac{1}{4 A_{0}^{2}}-\frac{1}{4}\right)\right| i\right\rangle=1.115 \frac{e^{2}}{\pi}\langle i|\beta m| i\rangle .
$$

We now turn to the modern $4 \mathrm{D}$ relativistically invariant formulation of the perturbation theory in the quantum field theory.

The self-energy operator (or mass operator) in the second-order of the perturbation theory is

$$
M(p)=-\frac{8 \pi i}{(2 \pi)^{4}} e^{2} \int \frac{2 m-\hat{p}+\hat{k}}{\left[(p-k)^{2}-m^{2}\right] k^{2}} d^{4} k .
$$

For the external electron line of Feynman diagram,

$$
p^{2}=p_{0}^{2}-\mathbf{p}^{2}=m^{2}, \quad \hat{p}|i\rangle=m|i\rangle .
$$

Introduce, according to [4], the notion of the limiting 4-impulse, $L^{2}=L_{0}^{2}-\mathbf{L}^{2}$, and perform the integration in (12) over the variable $k^{0}$ using the Feynman rule of pole bypass. For $L_{0} / m \gg$ 
1 the integration result is

$$
\begin{aligned}
M(p)= & -\frac{e^{2}}{4 \pi^{2}} \beta \int d \mathbf{k}\left[-\frac{|\mathbf{k}|-\alpha \mathbf{k}+\beta m}{|\mathbf{k}|\left(\tilde{p}_{0}+|\mathbf{k}|-p_{0}\right)\left(\tilde{p}_{0}-|\mathbf{k}|+p_{0}\right)}\right. \\
& +\frac{|\mathbf{k}|+\alpha \mathbf{k}-\beta m}{|\mathbf{k}|\left(\tilde{p}_{0}-|\mathbf{k}|-p_{0}\right)\left(\tilde{p}_{0}+|\mathbf{k}|+p_{0}\right)}+\frac{\left(p+\tilde{p}_{0}\right)-\alpha \mathbf{k}+\beta m}{\tilde{p}_{0}\left(\tilde{p}_{0}+|\mathbf{k}|+p_{0}\right)\left(\tilde{p}_{0}-|\mathbf{k}|+p_{0}\right)} \\
& \left.+\frac{\left(p-\tilde{p}_{0}\right)-\alpha \mathbf{k}+\beta m}{\tilde{p}_{0}\left(\tilde{p}_{0}+|\mathbf{k}|-p_{0}\right)\left(\tilde{p}_{0}-|\mathbf{k}|-p_{0}\right)}\right] .
\end{aligned}
$$

It can be shown that on the algebraic transformations the average of the expression in state $|i\rangle$ equals to that of expression (4) for $Y_{0}$ :

$$
\langle i|M(p)| i\rangle=\left\langle i\left|Y_{0}\right| i\right\rangle
$$

Hence, the spatial components of the introduced limiting impulse $L$ are equal, $L_{i}=q_{i}$. Since for free motion the only relativistic invariant is particle mass $m$, then $L^{2}=L_{0}^{2}-\mathbf{q}^{2}=C \cdot m^{2}$, where $C$ is a numerical factor. As $L_{0} / m \gg 1$, the factor $C$ should be also much higher than one. With $q$ varying as a function of the particle impulse, the time component $L_{0}$ should also vary accordingly, with the invariant $L^{2}$ remaining invariable.

Note that if the $4 \mathrm{D}$ integration in (12) is performed in the conventional manner, then expression (12) can be written as

$$
M(p)=\frac{e^{2}}{\pi} m\left[\frac{3}{4} \ln \left(1+\frac{L^{\prime 2}}{m^{2}}\right)+\frac{L^{\prime}}{2 m} \arctan \frac{m}{L^{\prime}}-\frac{1}{8} \frac{L^{\prime 2} / m^{2}}{1+L^{\prime 2} / m^{2}}\right] .
$$

At the limit $L^{\prime 2} \gg m^{2}$

$$
M(p) \approx \frac{e^{2}}{\pi} m\left[\frac{3}{4} \ln \left(\frac{L^{\prime 2}}{m^{2}}\right)+\frac{3}{8}\right],
$$

which coincides with a similar expression obtained in [4].

The components of impulse $L^{\prime}$ in (13) form the Euclidean 4 D sphere $\left(L^{\prime 2}=L_{0}^{\prime 2}+\mathbf{L}^{\prime 2}\right)$ because of the rotation of the path of integration over variable $k^{0}$ in the complex plane. Besides, the components of impulse $L^{\prime}$ differ from those of the previously introduced impulse $L$ because of the change in the domain of integration from the replacement of variables, $k-p \rightarrow k$ [4].

To determine $L^{\prime}$, once again use equality

$$
\langle i|M(p)| i\rangle=\left\langle i\left|Y_{0}(q)\right| i\right\rangle .
$$

Then,

$$
\frac{3}{4} \ln \left(1+\frac{L^{\prime 2}}{m^{2}}\right)+\frac{L^{\prime}}{2 m} \arctan \frac{m}{L^{\prime}}-\frac{1}{8} \frac{L^{\prime 2} / m^{2}}{1+L^{\prime 2} / m^{2}}=1,115 .
$$

The solution to equation (14) is $L^{\prime} / m=1.335 \approx 4 / 3, \quad L^{\prime 2} \approx 1.8 m^{2}$.

With consideration of the above-described regularization of the expression for the self-energy of free Dirac particle, the particle mass change due to the renormalization in the second order of the perturbation theory is

$$
\Delta m^{(2)}=\frac{e^{2}}{\pi} m\left(\frac{3}{2} \ln \left(A_{0}\right)+\frac{1}{4 A_{0}^{2}}-\frac{1}{4}\right)=1.115 \frac{e^{2}}{\pi} m .
$$


The re-normalized particle mass is

$$
m^{(2)}=m_{0}+\Delta m^{(2)}=m_{0}\left(1+1.115 \frac{e^{2}}{\pi}\right),
$$

where $m_{0}$ is the bare mass of the particle.

By setting the self energy of Dirac particle at rest, $\left\langle i\left|Y_{0}(|\mathbf{p}|=0)\right| i\right\rangle$, equal to electrostatic energy, $e^{2} / r_{e m}$, we obtain the electromagnetic radius estimate for non-relativistic particle: $r_{\text {em }} \approx 1 / 2 m$.

Given external fields $A^{\mu}(\mathbf{x})$, the integration limit $q$ and time component $L_{0}$ will depend on their magnitude with retained invariant $L^{2}$.

For weak external fields $A^{\mu}(\mathbf{x})$, where in self-energy expression (11) we can restrict ourselves to inclusion only of terms not higher than quadratic in the generalized particle impulse, the results of the calculation for the anomalous magnetic moment of the particle and Lamb shift of energy levels in the second order of the perturbation theory within the approach developed in this paper will be presented in the following publication.

In the presence of high external electromagnetic fields, electromagnetic radius $r_{e m}$ will reduce owing to changes of the integration limit $q$, thus ensuring quantum electrodynamics applicability up to ultra-short distances.

Of course, it would be desirable that $q\left(A^{\mu}(\mathbf{x})\right)$ be obtained from first principles in the future

Summarize the results of the paper.

1. An algorithm for regularization of self-energy expressions for Dirac particle satisfying the requirements of the relativistic and gauge invariance is proposed.

2. The expression for the upper integration limit $q$ is a relatively slowly varying function of particle impulse within the second-order formulas of the "old" perturbation theory for free motion.

For a particle at rest, $q=m$; for $p / m$ in the range from 0 to 1 ,

$$
q \approx m\left(1+0.00864 \frac{p^{4}}{m^{4}}-0.00217 \frac{p^{6}}{m^{6}}\right)
$$

for the ultra-relativistic case $|\mathbf{p}| \gg m, q \approx 1.5 m$.

It is shown for $4 \mathrm{D}$ perturbation theory upon introduction of limiting 4-impulse $L^{2}=L_{0}^{2}-\mathbf{L}^{2}$ that with a large magnitude of the time component, $L_{0} / m \gg 1$, the spatial values of $L_{i}$ are limited and are the same as the components of the introduced limits of integration $q_{i}$ : $\mathbf{L}^{2}=\mathbf{q}^{2}$.

For the conventional integration techniques for Feynman integrals in the second order of the perturbation theory the limiting integration impulse is evaluated: $L^{\prime 2}=L_{0}^{\prime 2}+\mathbf{L}^{\prime 2} \approx 16 \mathrm{~m}^{2} / 9$.

3. In the second order of the perturbation theory, the re-normalized mass of Dirac particle is

$$
m^{(2)}=m_{0}+\Delta m^{(2)}=m_{0}\left(1+1.115 \frac{e^{2}}{\pi}\right)
$$

where $m_{0}$ is the bare mass of the particle. 
4. The electromagnetic radius of particle at rest that can be found by equating the selfenergy and the electrostatic energy, $e^{2} / r_{e m}$, is approximately half the Compton wavelength, $r_{e m} \approx 1 / 2 m$.

\section{References}

[1] P.A.M. Dirac. Lectures on quantum field theory. Moscow, Mir Publishers, 1971.

[2] P.A.M. Dirac. Quantum mechanics principles. Moscow, Nauka Publishers, Editors-in-chief for physics and mathematics literature, 1979.

[3] V.P. Neznamov. Doklady Akademii Nauk, 1998, vol.1, p. 44-46.

[4] A.N.Akhiyezer, V.V.Berestetskiy. Quantum electrodynamics, Moscow, Nauka Publishers, Editors-in-chief for physics and mathematics literature, 1969. 